\title{
miR-3147 serves as an oncomiR in vulvar squamous cell cancer via Smad4 suppression
}

\author{
XIU-HUA YANG ${ }^{1}$ and FENG GUO ${ }^{2}$ \\ ${ }^{1}$ Department of Obstetrics, The First Affiliated Hospital of China Medical University, Shenyang, Liaoning 110001; \\ ${ }^{2}$ Department of Emergency, Shengjing Hospital of China Medical University, Shenyang, Liaoning 110004, P.R. China
}

Received September 3, 2017; Accepted March 1, 2018

DOI: $10.3892 / \mathrm{mmr} .2018 .8697$

\begin{abstract}
The incidence of vulvar squamous cell carcinoma (VSCC) has increased annually over the last decade. MicroRNAs (miRNAs/miRs) serve an important role in tumor progression and development. Our previous microarray studies have revealed that miR-3147 was overexpressed in VSCC. However, its function and underlying mechanism in VSCC remain unknown. In the present study, it was confirmed by reverse transcription-quantitative polymerase chain reaction that the expression of miR-3147 was markedly upregulated in VSCC tissues. The increased expression of miR-3147 was positively associated with the depth of invasion. The overexpression of miR-3147 resulted in the promotion of vulvar cancer cell proliferation, migration, invasion, G1/S progression and invasion-associated gene expression. miR-3147 may participate in the process of epithelial-mesenchymal transition and reduce the expressions of downstream target genes in the transforming growth factor- $\beta /$ Smad signaling pathway in A431 cells. The knockdown of Smad4 by small interfering RNA promoted malignant behaviours in A431 cells. In addition, miR-3147 regulated Smad4 by directly binding to its 3' untranslated region. In conclusion, the results indicated that miR-3147 may serve an oncogenic role in VSCC by targeting Smad4. miR-3147 may represent a novel potential therapeutic target marker for VSCC.
\end{abstract}

Correspondence to: Dr Xiu-Hua Yang, Department of Obstetrics, The First Affiliated Hospital of China Medical University, 155 Nanjing North Street, Shenyang, Liaoning 110001, P.R. China E-mail: yangxiuhuajing@hotmail.com

Abbreviations: VSCC, vulvar squamous cell carcinoma; miRNA, microRNA; EMT, epithelial-mesenchymal transition; WHO, World Health Organization; FIGO, International Federation of Gynecology and Obstetrics; UICC, Union for International Cancer Control; cDNA, complementary DNA; MMP-2, matrix metalloproteinase 2; DMSO, dimethylsulfoxide; NCCN, National Comprehensive Cancer Network; OS, overall survival; HNSCC, head and neck squamous cell carcinomas

Key words: vulvar squamous cell carcinoma, microRNA-3147, Smad4, EMT, transforming growth factor- $\beta$

\section{Introduction}

Malignant tumors of the vulva represent a very small number of female genital malignancies, $80-90 \%$ of vulvar cancers are vulvar squamous cell carcinomas (VSCCs). There will be about 6020 new patients in 2017, and 1150 people are expected to die. The incidence is increasing by $0.6 \%$ annually in the last 10 years (1). Women with distant metastases $(5 \%)$ and local diffusion (31\%) have bad prognosis. The 5 year survival rates were 17 and $57 \%$, respectively (1). Thus it is of important clinical essentiality to research the occurrence and development of VSCC. In the present case, many scholars have begun to focus on the microRNAs (miRNAs).

miRNAs prevent translation or promote mRNA degradation (2). miRNA plays a key role in the carcinogenesis and evolution by accommodating epithelial-mesenchymal transition (EMT), oncogenic signaling pathways and metastasis (3). miRNAs repress target gene expression and usually perform important functions in cancers.

Based on our previous findings (4), miR-3147 was markedly increased in VSCC tissues, but the underlying mechanism is still unknown. Smad4 is responsible for regulating the TGF- $\beta$ /Smad signaling pathway and it was downregulated in VSCC samples (4). Based on the target prediction program miRanda, Smad4 is a target gene for miR-3147, but the association between miR-3147 and Smad4 in VSCC requires further investigation. In the research, we performed functional studies to find the roles of miR-3147 and Smad4 in VSCC. Smad4 was confirmed to be a target gene of miR-3147.

\section{Materials and methods}

Experimental samples. Twenty VSCC and adjacent non-dysplastic tissues were obtained from the patients admitted to the Department of Gynaecology at our hospital between 2010 and 2017. Every sample of VSCC was negative in HPV, and Linear Array HPV Genotyping (Roche Applied Science, Pleasanton, CA, USA) was used for the approach HPV testing. According to the standard of World Health Organization (WHO), the diagnosis in histopathology was conducted. The grade of tumor was determined according to the new version of the International Federation of Gynecology and Obstetrics (FIGO) system published in 2010; the stage of tumor was identified by the 7 th TNM categorization of the Union for 
International Cancer Control (UICC). For the stages of tumors that diagnosed before 2009, they were identified again based the latest version. The frozen specimens were microscopically identified by two pathologists. Clinicopathological data were retrospectively collected. Patients without preoperative radiotherapy or chemotherapy were chosen. The research was performed based on the Helsinki declaration. Prior to surgery, all subjects provided written informed consent to participate in the study. The Ethics Committee of the First Affiliated Hospital of China Medical University (Liaoning, China) approved the present study.

Cell culture and transfection. Acquirement of the A431 cell line was made from ATCC, in addition to culturing in RPMI 1640 with $10 \%$ fetal bovine serum at a temperature of $37^{\circ} \mathrm{C}$ and a humid atmosphere of $5 \% \mathrm{CO}_{2}$. Using the Dharmacon miRIDIAN miR-3147 mimics (miR-3147), the transfection of the cells was carried out, together with the negative control (Thermo Fisher Scientific, Inc., Waltham, MA, USA) at an eventual amounting to be $100 \mathrm{nmol} / \mathrm{l}$. A small interfering RNA targeting Smad4 (siR-Smad4; sc-29,484) was attained from Santa Cruz Biotechnology, Inc. (Dallas, TX, USA). Transfections were conducted with the help of Lipofectamine 2000 in accordance with the protocol of the manufacturer.

Reverse transcription-quantitative polymerase chain reaction $(R T-q P C R)$. Total RNA extraction was conducted by making use of TRIzol reagent (Takara Bio, Inc., Shiga, Japan) in accordance with the guidelines of the manufacturer. Stem-loop RT-PCR was performed for the purpose of testing the miR-3147 expression (5). Complementary DNA (cDNA) synthesis was conducted according to a Gene Amp PCR System 9700 (Applied Biosystems; Thermo Fisher Scientific, Inc.). We calculated the mRNA expressions with the $2^{-\Delta \Delta C q}$ approach. Furthermore, the primers for miRNAs and mRNAs are shown (Table I).

Western blotting. Cells were harvested, together with the calculation of protein concentration through the use of a protein assay kit (Bio-Rad Laboratories, Inc., Hercules, CA, USA). Three days subsequent to the transfection with the miR-3147 mimics or siRNA-Smad4, the total protein was obtained (6). In respect of immunoblotting, the membrane was incubated with antibodies against E-cadherin (1:500, ab15148; Abcam, Cambridge, UK), N-cadherin (1:500, ab18203; Abcam), Vimentin (1:500, ab24525; Abcam), matrix metalloproteinase 2 (MMP-2; 1:300; BIOSS, Beijing, China), MMP-9 (1:300; BIOSS) or GAPDH (1:10,000, ab181602; Abcam). Rinsing of the membrane was performed, followed by incubation with anti-mouse or anti-rabbit $\operatorname{IgG}(\mathrm{H}+\mathrm{L})$-HRP conjugate (1:10,000; Invitrogen; Thermo Fisher Scientific, Inc.) antibody. We made use of the Image J software (National Institute of Health, Bethesda, MD, USA) in order to analyze the results.

MTT test. Cell proliferation assay was assessed by MTT test. Ninety-six well plates were seeded with $5 \times 10^{4}$ cells per well. At the appointed time after transfection $(0,48$, and 96 h), $20 \mu$ l MTT (Sigma-Aldrich, St. Louis, MO, USA) was put into the well, followed by the incubation of the cells at a temperature of $37^{\circ} \mathrm{C}$ for a period of $4 \mathrm{~h}$. The liquid was abandoned and then we put $150 \mu 1$ dimethylsulfoxide (DMSO; Sigma-Aldrich) to lysis the formazan. Measurement of the optical density was also taken at $490 \mathrm{~nm}$ by using a microplate spectrophotometer.

Cell migration and invasion. For the purpose of testing cell migration, a Transwell test was conducted. $48 \mathrm{~h}$ of time following the transfection, addition of $5 \times 10^{4}$ cells was made into the above chambers of plates by an untreated membrane. Subsequent to $24 \mathrm{~h}$ of incubation, addition of $4 \%$ paraformaldehyde was made into the chambers, followed by a fixation using the crystal violet. We carried out the calculations of the cells, which passed all across the membrane. As for the invasion experiments, Matrigel (BD Biosciences, Franklin Lakes, NJ, USA) was used on the above chamber. The other mechanisms possessed similarities with the migration experimentation. All the tests were repeated in triplicate.

Distribution of cell cycle. More than $1 \times 10^{4}$ cells were trypsinized, collected, washed twice, as well as positioned in $70 \%$ ethanol for a time period of $24 \mathrm{~h}$. The cells were washed twice with PBS, following the incubation using $400 \mu \mathrm{l}$ RNase $(0.25 \mathrm{mg} / \mathrm{ml})$ at a temperature of $37^{\circ} \mathrm{C}$ for $1 \mathrm{~h}$, proceeding to treatment with propidium iodide $(40 \mu \mathrm{g} / \mathrm{ml})$. Determination of the cell cycle distribution was made through flow cytometry. The experiments were conducted for three times.

Luciferase reporter assay. The fragment of the human Smad4 with (wild-type) or without (mutant) the miR-3147 binding site at the 3'-untranslated region (3'-UTR) was cloned and inserted into the pGL3-basic luciferase report plasmid (Promega Corporation, Madison, WI, USA) to generate luciferase reporter vectors, Smad4 3'-UTR-wt and Smad4 3'-UTR-mut. The $293 \mathrm{~T}$ cells were treated in 96 -well plates at 5,000 cells per well and permitted to stay for $24 \mathrm{~h}$ prior to transfection. Then, transfection of the miR-3147 mimics or miR-NC was performed into the 293T cells with the use of Lipofectamine 2000 with 100 ng of Smad4 3'-UTR-wt or Smad4 3'-UTR-mut, in addition to $10 \mathrm{ng}$ of pRL-TK Renilla plasmid (Promega Corporation). Following the incubation for a time period of $48 \mathrm{~h}$, determination of the luciferase activities was performed with the help of a Dual Luciferase Reporter system (Promega Corporation).

Statistical method. The presentation of the data has been made as the means \pm SD. Furthermore, we made use of the SPSS 19.0 software (SPSS, Inc., Chicago, IL, USA) for the purpose of analyzing the findings. A P-value of $<0.05$ was thought to be statistically significant. Analysis of the significance of differences in the mean values was carried out with the application of Student's t-test. The use of a two-sided Fisher's exact test was made for the purpose of determining the association between the expression level of miR-3147 and clinicopathological data.

\section{Results}

RT-qPCR analysis. A RT-qPCR analysis of miR-3147 in VSCC as well as surrounding non-dysplastic tissues was conducted. The expression of miR-3147 was significantly increased in VSCC tissues ( $\mathrm{P}=8.67 \times 10^{-5}$; Fig. 1). 
Table I. Primers used in reverse transcription-quantitative polymerase chain reaction.

\begin{tabular}{lll} 
Gene & \multicolumn{1}{c}{ Forward primers (5'-3') } & \multicolumn{1}{c}{ Reverse primers $\left(5^{\prime}-3^{\prime}\right)$} \\
\hline U6 & CTCGCTTCGGCAGCACA & AACGCTTCACGAATTTGCGT \\
miR-3147 & GGUUGGGCAGUGAGGAGGGUGUGA & TACGAGGTTGGGCAGTGAGGAGGGTGT \\
Smad4 & CGCTTTTGTTGGGTCAACT & CCCAAACATCACCTTCACCTC \\
E-cadherin & TGCTGTTTCTGGTTTCTGTTGG & CTTCTCCGTATTCTCCTCCCT \\
N-cadherin & TTTGGGGAGGGGTAAAAGTTC & AAGAAACAGGCCACCCCTTT \\
Vimentin & CGGTTGAGACCAGAGATGGA & TGCTGGTACTGCACTGTTGG \\
P21 & TGTCCGTCAGAACCCATG & TGGGAAGGTAGAGCTTGG \\
PAI-1 & GAGACAGGCAGCTCGGATTC & GGCCTCCCAAAGTGCATTAC \\
Bax & ATGGACGGGTCCGGGGAGCAG & CATGATGGTTCTGATCAGT \\
Bim & GCCTTCAACCACTATCTCA & ATCCAGCTCGGTGTCTTCT \\
GAPDH & AAGGTGAAGGTCGGAGTCAAC & GGGTCATTGATGGCAACAATA
\end{tabular}

miR, microRNA; PAI-1, plasminogen activator inhibitor-1; Bax, B-cell lymphoma 2-associated X protein; Bim, B-cell lymphoma-2-like protein 11 .

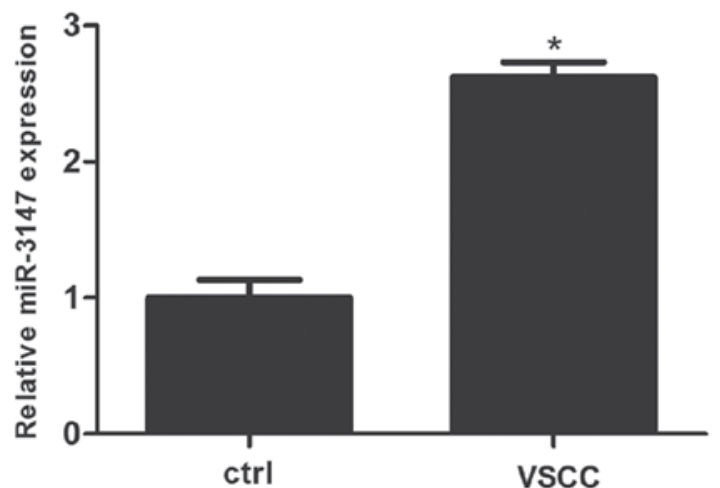

Figure 1. Relative expression of miR-3147 in VSCC and adjacent non-dysplastic tissues. miR-3147 was upregulated in VSCC. ${ }^{*} \mathrm{P}<0.05$ vs. ctrl. VSCC, vulvar squamous cell carcinoma; miR, microRNA; Ctrl, control.

Relationship between miR-3147 and clinicopathological parameters. The 75 th percentile of the $2^{-\Delta \Delta \mathrm{Ct}}$ values was considered to be the cut-off value for tissues with low or high expressions of miR-3147 (7). The expression level of miR-3147 was not related to age, tumor differentiation, vascular invasion, FIGO stage, tumor size and lymph node metastasis, but was positively related to the depth of invasion ( $\mathrm{P}=0.023$; Table II). The results showed that the upregulation of miR-3147 contributes to the invasion of VSCC.

Effects of miR-3147 on vulvar carcinoma in vitro. In comparison with the negative control, the proliferation rate of the cells that had undergone transfection with the miR-3147 mimics amounted to be significantly higher at $96 \mathrm{~h}(\mathrm{P}=0.007$; Fig. 2A). Selection of MMP-2 as well as MMP-9 was made for the purpose of testing if miR-3147 was capable of affecting the genes associated with tumor invasion. MMP-2 and MMP-9 were increased after transfection (Fig. 2B). For the fact that Smad 4 is termed as a crucial mediator in TGF- $\beta$ signal pathway, the inhibition of Smad4 by miR-3147 is most likely to decrease the expressions of downstream target genes in this pathway. Our results displayed that miR-3147 transfection resulted into the inhibition of the mRNA levels of p21 ( $\mathrm{P}=0.007)$, PAI-1 $(\mathrm{P}=0.011)$, $\mathrm{Bax}(\mathrm{P}=0.007)$ and $\mathrm{Bim}(\mathrm{P}=0.002$; Fig. 2C), indicating miR-3147 could regulate Smad4-mediated signaling pathway. The miR-3147 mimics markedly increased the migration $(\mathrm{P}=0.002$; Fig. 2D) and invasion $(\mathrm{P}=0.015$; Fig. $2 \mathrm{E})$ of A431 cells. Cell cycle results revealed the fact that the cells in the G1 phase were notably dropped from $62.34 \pm 1.62 \%$ to $57.87 \pm 1.78 \%(\mathrm{P}=0.032$; Fig. $2 \mathrm{~F})$. In addition, the cells in the $\mathrm{S}$ phase were remarkably added from $24.84 \pm 1.07 \%$ to $27.70 \pm 0.94 \%$ at $48 \mathrm{~h}(\mathrm{P}=0.004$; Fig. $2 \mathrm{~F})$.

Effects of Smad4 on vulvar carcinoma in vitro. The siR-Smad4 was performed to discuss the effect of Smad4 in vulvar cancer. Smad4 knockdown resulted into a surprising promotion of the cell proliferation $(\mathrm{P}=0.001 ;$ Fig. $3 \mathrm{~A})$. siR-Smad4 transfection led to the downregulation of the mRNA as well as protein expression levels of Smad4 by $47.67 \pm 5.51 \%\left(\mathrm{P}=1.18 \times 10^{-4}\right.$; Fig. 3B) and $40.00 \pm 1.73 \%$ ( $\mathrm{P}=7.07 \times 10^{-5}$; Fig. $3 \mathrm{C}$ ), respectively. Smad4 knockdown also markedly increasd migration $(\mathrm{P}=0.003$; Fig. 3D) and invasive ability ( $\mathrm{P}=0.011$; Fig. $3 \mathrm{E})$, in addition to decreasing cells in the G1 stage from $60.24 \pm 0.83 \%$ prior to knockdown to $58.05 \pm 1.04 \%$ at $48 \mathrm{~h}$ subsequent to the knockdown $(\mathrm{P}=0.003$; Fig. 3F).

The effect of miR-3147 in EMT. The transfection of the miR-3147 mimics into A431 cells was performed. After 4 days, no obvious change in the shape of cells was observed. miR-3147 transfection downregulated E-cadherin protein expression, in addition to upregulating the protein expressions of $\mathrm{N}$-cadherin as well as Vimentin (Fig. 2B). The findings revealed the fact that miR-3147 could take part in the mechanism of EMT in vulvar cancer.

miR-3147 targets Smad4. We searched the target prediction website miR and a (http://www.microrna.org/microrna/ home.do) and predicted that the miR-3147 promoter contains an Smad4 binding site (Fig. 4A). miR-3147 transfection reduced Smad4 mRNA expression by $33.0 \pm 7.0 \%$ after $48 \mathrm{~h}(\mathrm{P}=0.001$; Fig. 4B). The change of Smad4 protein was consistent with that 
Table II. Associations between the expression of microRNA-3147 and clinical pathology.

\begin{tabular}{|c|c|c|c|c|}
\hline Variable & $\begin{array}{c}\text { Number of } \\
\text { cases (n) }\end{array}$ & $\begin{array}{l}\text { Low } \\
\text { expression (n) }\end{array}$ & $\begin{array}{c}\text { High } \\
\text { expression (n) }\end{array}$ & P-value \\
\hline Age (years) & & & & 0.178 \\
\hline $25-69$ & 10 & 7 & 3 & \\
\hline $70-85$ & 10 & 4 & 6 & \\
\hline Differentiation & & & & 0.961 \\
\hline Well & 10 & 4 & 6 & \\
\hline Moderate & 7 & 3 & 4 & \\
\hline Poor & 3 & 1 & 2 & \\
\hline Vascular invasion & & & & 0.531 \\
\hline Yes & 3 & 1 & 2 & \\
\hline No & 17 & 9 & 8 & \\
\hline Lymph node metastasis & & & & 0.436 \\
\hline No & 15 & 6 & 9 & \\
\hline Yes & 5 & 3 & 2 & \\
\hline FIGO staging & & & & 0.286 \\
\hline I & 10 & 2 & 8 & \\
\hline II & 7 & 4 & 3 & \\
\hline III & 3 & 1 & 2 & \\
\hline Tumor diameter (cm) & & & & 0.989 \\
\hline $0.3-2.5$ & 10 & 3 & 7 & \\
\hline $2.6-4.0$ & 7 & 2 & 5 & \\
\hline $4.1-20.0$ & 3 & 1 & 2 & \\
\hline Depth of invasion (mm) & & & & $0.023^{\mathrm{a}}$ \\
\hline $0.0-4.0$ & 10 & 2 & 8 & \\
\hline $4.1-8.0$ & 7 & 6 & 1 & \\
\hline $8.1-40.0$ & 3 & 2 & 1 & \\
\hline
\end{tabular}

${ }^{a} \mathrm{P}<0.05$. FIGO, International Federation of Gynecology and Obstetrics.

of mRNA ( $\mathrm{P}=2.75 \times 10^{-4}$; Fig. 4C). Silencing Smad4 promoted the expression level of miR-3147 ( $\mathrm{P}=0.001$; Fig. 4D). Dual-luciferase reporter assays results indicated that the expression of luciferase reporter gene linked with part sequence of the 3'UTR of Smad4 gene was downregulated by the overexpression of miR-3147 in A431 cells ( $\mathrm{P}=0.004$; Fig. 4E), and the downregulation behavior was abolished when the miR-3147 binding site in 3'UTR of Smad4 was mutated ( $\mathrm{P}=0.571$; Fig. 4E).

\section{Discussion}

VSCC is a relatively rare disease. Standard therapy for VSCC has been described in the recently developed National Comprehensive Cancer Network (NCCN) compendium for VSCC. Nonetheless, advanced VSCC carries a poor prognosis and it is less responsive to cytotoxic agents than other solid tumors. Patients with advanced vulvar carcinoma experience significantly shorter overall survival (OS) durations than those with other metastatic or recurrent solid tumors treated with novel phase I therapeutics (8). Because of this poor prognosis in advanced disease, the development of satisfactory improvements is warranted and necessary $(9,10)$.
miRNA is widely studied in many fields of medicine including cancers. The identification of 79 miRNAs was made by de Melo Maia et al (11), which revealed surprisingly varied expression levels in vulvar cancer in comparison with control speciments. In addition, they also explored some links between special miRNAs and the clinical pathological data (11). Furthermore, a miRNA sponge was also verified by them, which was most likely to appear as an efficient method for diagnosing and treating this cancer (12). It has been reported that miR-223-5p works as an oncogene by targeting p63 in vulvar cancer (13). miR-590-5p drives promotion of cellular malignant conduct with the help of the target gene TGF $\beta$ RII in VSCC (4). In this study, we discussed the mechanism of miR-3147 in VSCC.

There were relatively fewer studies of miR-3147. It has been reported to be upregulated in cervical squamous cancer (14) and melanoma (15), while the specific mechanism of action of miR-3147 in tumors has not been studied. In our study, 20 fresh VSCC tissues were collected to test the expression of miR-3147 by RT-qPCR. The expression of miR-3147 was markedly increased in VSCC tissues and miR-3147 expression was positively related to the depth of invasion. We next increased 
A

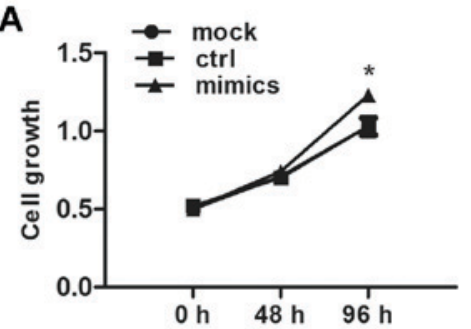

D

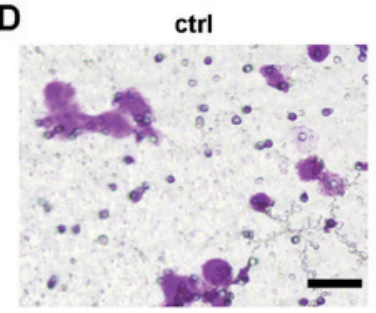

E

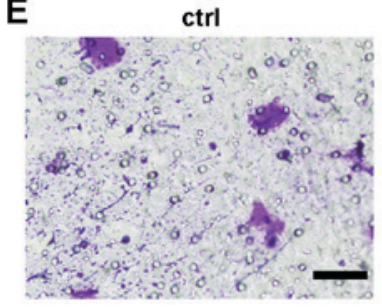

F

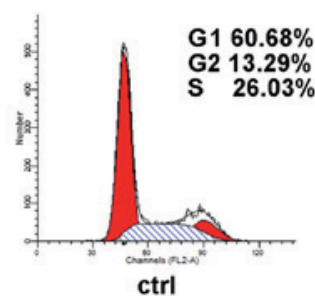

B
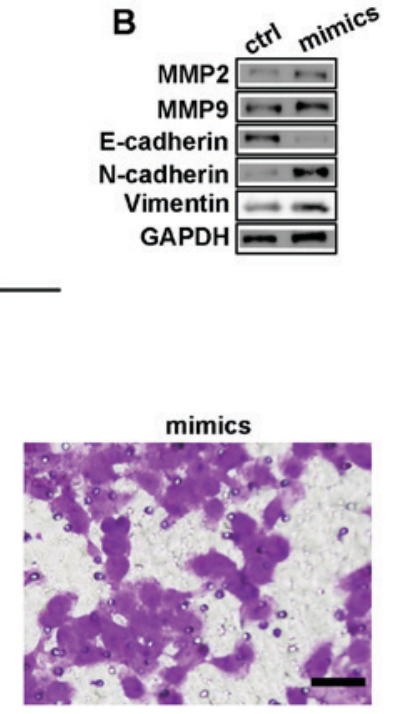

mimics
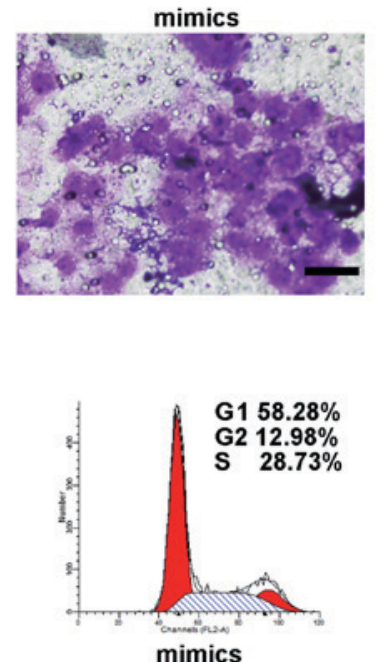
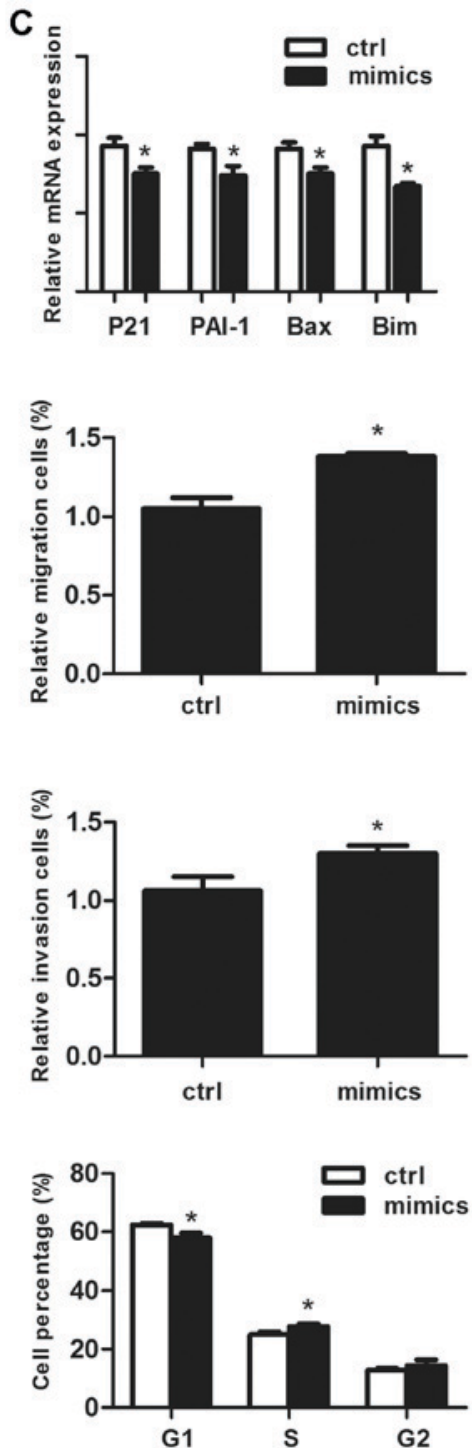

Figure 2. Effects of miR-3147 on vulvar carcinoma in vitro. Overexpression of miR-3147 (A) increased the rate of growth, (B) promoted EMT and increased levels of MMP-2 and MMP-9, (C) decreased downstream target genes of Smad4, (D) increased migration, (E) increased invasion (scale bars, $200 \mu$ m) and (F) promoted G1/S progression. * $\mathrm{P}<0.05$ vs. ctrl. miR, microRNA; Ctrl, control; EMT, epithelial-mesenchymal transition; MMP, matrix metalloproteinase; Bax, B-cell lymphoma 2-associated X protein; Bim, B-cell lymphoma-2-like protein 11; PAI-1, plasminogen activator inhibitor-1.

miR-3147 by transfection with the miR-3147 mimics in VSCC cell line A431. miR-3147 increased vulvar cancer cell proliferation, migration, invasion and G1/S progression (Fig. 2). These results reinforce that miR-3147 functions as an oncogene in vulvar carcinoma.

When classify the VSCCs, we can summarize two different kinds. The first one is HPV-associated, which accounts for 20 to $50 \%$ in the whole cases. The second one is HPV-independent. In epidemiology, clinics, pathology and molecular mechanism, all of them have different features. While A431 cells are HPV negative, we studied the expression of miR-3147 in HPV negative samples and A431 cells to avoid viral interference in miRNA pathways. As we know, no information about miR-3147 and the infection of HPV has been reported in other tumors. 25 different expressions of miRNAs have been found between HPV-positive vulvar cancers and HPV-negative vulvar cancers (11). By changing the expressions of miRNAs, the HPVs have oncogenic properties. This conclusion has been proved through some HPV-related researches such as cervical cancer. Researchers thought that there was a connection between cancer progression and the expressions of miR-92a and miR-378 in HPV-positive cervical cancer tissue samples (16). At the same time, there is a report showing that the overexpression of miR-155 was linked to the increasing danger of cervical cancer in HPV E6/E7 mRNA positive tissues (17). There are lot of factors that can distinguish HPV-positive head and neck squamous cell carcinomas (HNSCC) patients from HPV-negative HNSCC patients. For example, a panel includes miR-9, miR-134, miR-196b, miR-210 and miR-455 (18).

Smad4 poses to be a vital regulatory factor in the TGF- $\beta /$ Smad signaling pathway (19), and the downregulation of Smad4 is also indicative of advanced tumor stage and a worse prognosis (20). Smad4 performs the function of a tumor suppressor gene in a great number of cancers, for instance pancreatic cancer (21), colorectal cancer (22), lung cancer (23), gastric adenocarcinoma (24), cervical cancer (25), HNSCC (26) and breast cancer (27). Smad4 was reported to be oncogenic in other cancers such as hepatocellular carcinoma (28), suggesting 

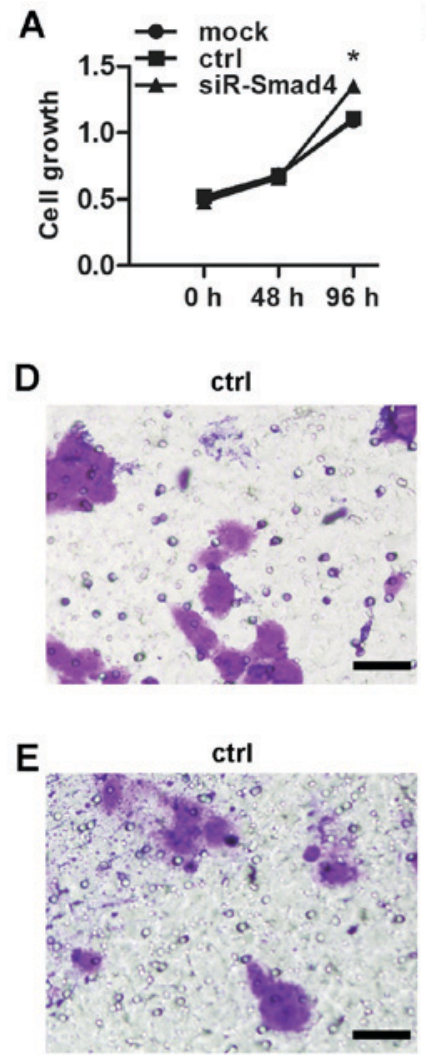

$\mathbf{F}$

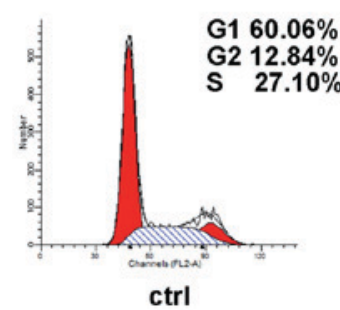

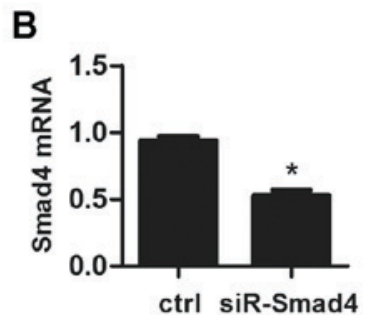
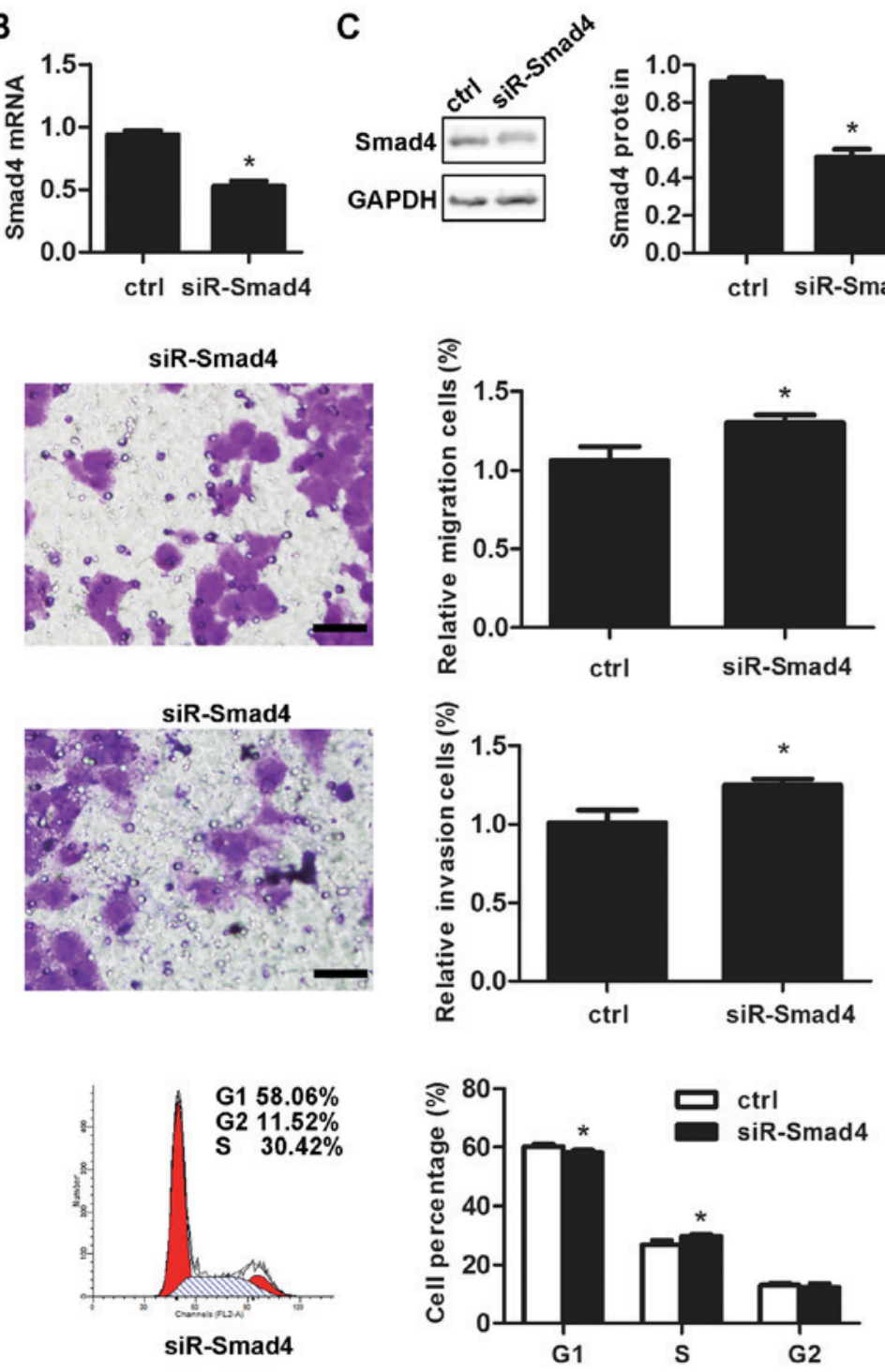

Figure 3. Effects of Smad4 on vulvar carcinoma in vitro. Downregulated expression of Smad4 (A) increased the rate of growth, (B) decreased Smad4 mRNA expression, (C) decreased Smad4 protein expression, (D) increased migration, (E) increased invasion (scale bars, $200 \mu \mathrm{m})$ and (F) promoted G1/S progression. ${ }^{*} \mathrm{P}<0.05$ vs. ctrl. siR, small interfering RNA; Ctrl, control.

that its role might be cell-context-dependent. Smad4 has been validated as a direct target of several oncogenic miRNAs, including miR-224 (29), miR-20a-5p (30) miR-1285 (31), miR-210 (25) and miR-130a/301a/454 (32). Consistent with these results, our study found the down-expression of Smad4 in A431 cells can simulate the oncogenic role of miR-3147 (Fig. 3), revealing that Smad4 acts as a tumor suppressor in VSCC. TGF- $\beta /$ Smad signaling pathway as well as miRNAs participate in various cellular processes. However, the association between the TGF- $\beta$ /Smad signaling pathway and miR-3147 in VSCC is not fully understood. We found the miR-3147 mimics could suppress the mRNA expressions of downstream target genes of Smad4, indicating that miR-3147 negatively regulates the TGF- $\beta$ signaling pathway via the suppression of Smad4.

Cell migration and invasion are complicated biological processes that have key effects on the progression of cancer. EMT has a key role in the progression and metastasis of tumors. Cancer cells are capable of spreading and invading other tissues when they experience EMT (33). We increased miR-3147 in A431 and tested the expressions of key molecules in EMT.
E-cadherin decreased but N-cadherin and Vimentin increased, revealing that the miR-3147 mimics could promote EMT (Fig. 2B). Current studies have confirmed that certain MMPs promote the progression of EMT. We also identify this view. We found that miR-3147 promoted MMP-2 and MMP-9 expression (Fig. 2B).

A prediction was validated by the application of the dual luciferase reporter assay, which suggested that the miR-3147 promoter sequence possesses a Smad4 binding site. The down-expression of Smad4 led to the promotion of miR-3147 mRNA expression (Fig. 4D). Furthermore, the miR-3147 mimics exhibited a decrease in the mRNA as well as protein expressions of Smad4 (Fig. 4B and C) that evidences the fact that miR-3147 targets Smad4 directly in VSCC.

In this article, we pointed out that miR-3147 can play as an oncomiR by promoting vulvar cancer cells invasion and metastasis. In this context, we can argue that miR-3147 has a great potential on treatment. The restriction of this oncomiR in high miR-3147/low Smad4 patients via synthetic miRNA silencing mechanisms including antimiR nucleotides might 

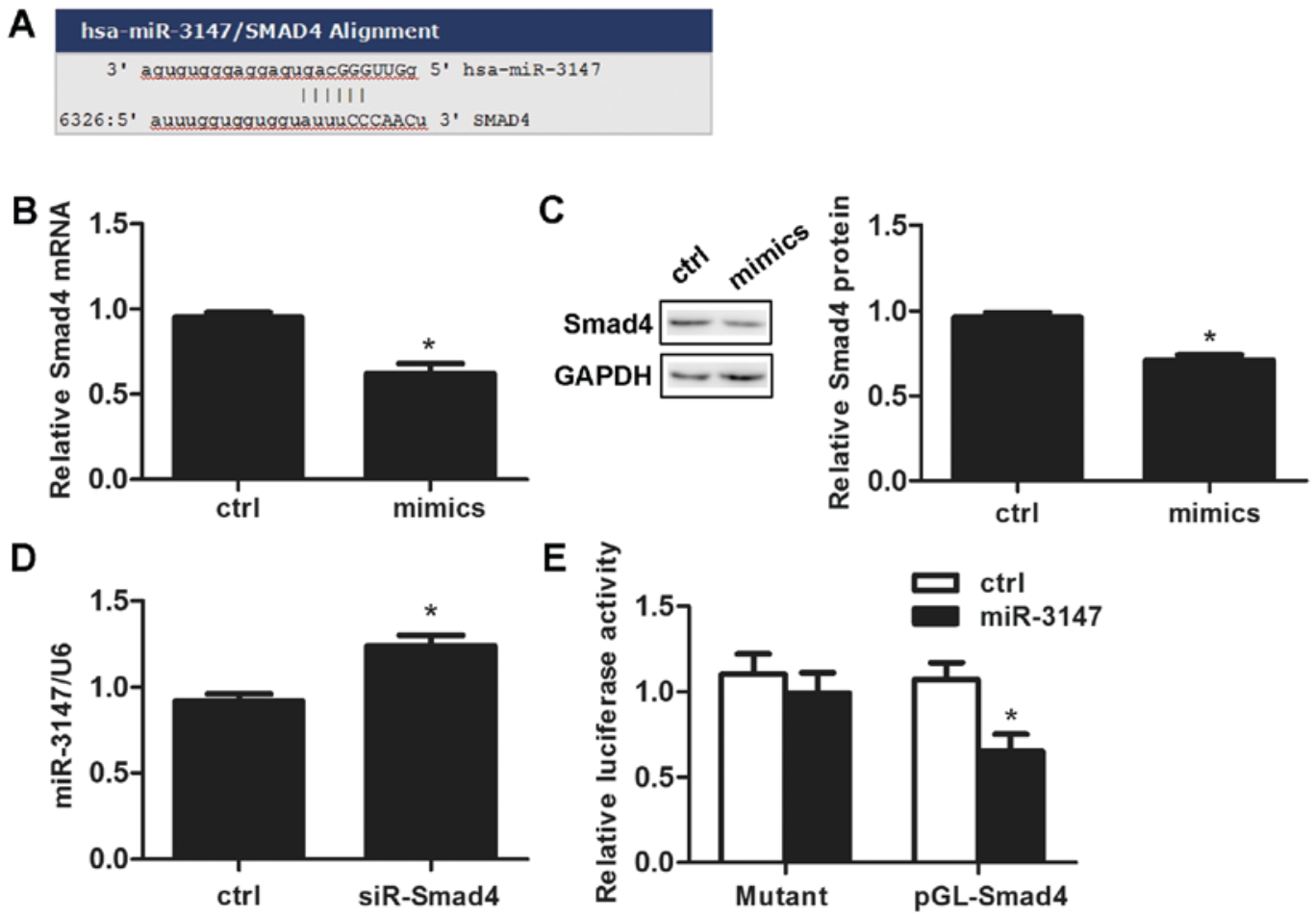

Figure 4. miR-3147 downregulated Smad4. (A) The miRanda website predicted that the Smad4 mRNA 3' untranslated region contained miR-3147 binding sites. (B) Overexpression of miR-3147 increased Smad4 mRNA. (C) Overexpression of miR-3147 decreased Smad4 protein levels. (D) Reduced expression of Smad4 increased miR-3147 mRNA levels. (E) Luciferase reporter assays confirmed that Smad4 was the target of miR-3147. * P<0.05 vs. ctrl. miR, microRNA; Ctrl, control.

be an equipment for inhibiting offense from the tumor. In addition, it might become a chance for the therapy of VSCC. Further studies should be followed in this direction. The goals are not only to examine if our findings can be applied in a wider range, but also to build in vitro and in vivo inhibition models for miR-3147 in VSCC.

In conclusion, we transfected the miR-3147 mimics and siR-Smad4 into A431 cells whereby we discovered that introducing an increase in the expression of miR-3147 and a decrease in the expression of Smad4 could result into promoting vulvar cancer cell proliferation, migration, invasion and G1/S progression. miR-3147 performs the function of an oncogene for playing a key biological function by targeting Smad4 in VSCC. As for the etiology and progression of vulvar carcinoma, miR-3147/Smad4 may be a new research direction. For example, making detection of the expression levels of miR-3147 or Smad4 in the blood or tissues of patients is likely to be used for early detection and prognostic evaluation. Therefore, our study may contribute to the diagnosis and treatment of VSCC in the future.

\section{Acknowledgements}

Not applicable.

\section{Funding}

No funding was received.

\section{Availability of data and materials}

The datasets used during the current study are available from the corresponding author on reasonable request.

\section{Authors' contributions}

XHY analyzed and interpreted the patients' data, and was a major contributor in writing the manuscript. FG performed the histological examination of the tissues. All authors have read and approved the final manuscript.

\section{Ethics approval and consent to participate}

The present study was approved by the Ethics Committee of the First Affiliated Hospital of China Medical University. All subjects provided written informed consent to participate in the study.

\section{Consent for publication}

All subjects provided written informed consent.

\section{Competing interests}

The authors declare that they have no competing interests.

\section{References}

1. Siegel RL, Miller KD and Jemal A: Cancer statistics, 2017. CA Cancer J Clin 67: 7-30, 2017.

2. Sha D, Lee AM, Shi Q, Alberts SR, Sargent DJ, Sinicrope FA and Diasio RB: Association study of the let-7 miRNA-complementary site variant in the $3^{\prime}$ untranslated region of the KRAS gene in stage III colon cancer (NCCTG N0147 Clinical Trial). Clin Cancer Res 20: 3319-3327, 2014.

3. Hiramoto H, Muramatsu T, Ichikawa D, Tanimoto K, Yasukawa S, Otsuji E and Inazawa J: miR-509-5p and miR-1243 increase the sensitivity to gemcitabine by inhibiting epithelial-mesenchymal transition in pancreatic cancer. Sci Rep 7: 4002, 2017. 
4. Yang $\mathrm{XH}$ and $\mathrm{Wu} \mathrm{X}$ : miRNA expression profile of vulvar squamous cell carcinoma and identification of the oncogenic role of miR-182-5p. Oncol Rep 35: 398-408, 2016.

5. Chen C, Ridzon DA, Broomer AJ, Zhou Z, Lee DH, Nguyen JT, Barbisin M, Xu NL, Mahuvakar VR, Andersen MR, et al: Real-time quantification of microRNAs by stem-loop RT-PCR. Nucleic Acid Res 33: e179, 2005.

6. Li Y, Wang F, Xu J, Ye F, Shen Y, Zhou J, Lu W, Wan X, Ma D and Xie X: Progressive miRNA expression profiles in cervical carcinogenesis and identification of HPV-related target genes for miR-29. J Pathol 224: 484-495, 2011.

7. Chen ZL, Zhao XH, Wang JW, Li BZ, Wang Z, Sun J, Tan FW, Ding DP, Xu XH, Zhou F, et al: microRNA-92a promotes lymph node metastasis of human esophageal squamous cell carcinoma via E-cadherin. J Biol Chem 286: 10725-10734, 2011.

8. Fu S, Shi N, Wheler J, Naing A, Janku F, Piha-Paul S, Gong J, Hong D, Tsimberidou A, Zinner R, et al: Characteristics and outcomes for patients with advanced vaginal or vulvar cancer referred to a phase I clinical trials program: The MD Anderson Cancer Center experience. Gynecol Oncol Res Pract 2: 10, 2015.

9. Economopoulou P, Agelaki S, Perisanidis C, Giotakis EI and Psyrri A: The promise of immunotherapy in head and neck squamous cell carcinoma. Ann Oncol 27: 1675-1685, 2016.

10. Tewari KS, Sill MW, LongHJ III, Penson RT, Huang H, Ramondetta LM, Landrum LM, Oaknin A, Reid TJ, Leitao MM, et al: Improved survival with bevacizumab in advanced cervical cancer. N Engl J Med 370: 734-743, 2014.

11. de Melo Maia B, Lavorato-Rocha AM, Rodrigues LS, Coutinho-Camillo CM, Baiocchi G, Stiepcich MM, Puga R, de A Lima L, Soares FA and Rocha RM: microRNA portraits in human vulvar carcinoma. Cancer Prev Res (Phila) 6: 1231-1241, 2013.

12. de Melo Maia B, Ling H, Monroig P, Ciccone M, Soares FA, Calin GA and Rocha RM: Design of a miRNA sponge for the miR-17 miRNA family as a therapeutic strategy against vulvar carcinoma. Mol Cell Probes 29: 420-426, 2015.

13. de Melo Maia B, Rodrigues IS, Akagi EM, Soares do Amaral N, Ling H, Monroig P, Soares FA, Calin GA and Rocha RM: MiR-223-5p works as an oncomiR in vulvar carcinoma by TP63 suppression. Oncotarget 7: 49217-49231, 2016.

14. Chen J, Yao D, Li Y, Chen H, He C, Ding N, Lu Y, Ou T, Zhao S, Li L and Long F: Serum microRNA expression levels can predict lymph node metastasis in patients with early-stage cervical squamous cell carcinoma. Int J Mol Med 32: 557-567, 2013.

15. Stark MS, Tyagi S, Nancarrow DJ, Boyle GM, Cook AL, Whiteman DC, Parsons PG, Schmidt C, Sturm RA and Hayward NK: Characterization of the melanoma miRNAome by deep sequencing. PLoS One 5: e9685, 2010

16. Wang X, Wang HK, Li Y, Hafner M, Banerjee NS, Tang S, Briskin D, Meyers C, Chow LT, Xie X, et al: microRNAs are biomarkers of oncogenic human papillomavirus infections. Proc Natl Acad Sci USA 111: 4262-4267, 2014.

17. Park S, Eom K, Kim J, Bang H, Wang HY, Ahn S, Kim G, Jang H, Kim S, Lee D, et al: MiR-9, miR-21 and miR-155 as potential biomarkers for HPV positive and negative cervical cancer. BMC Cancer 17: 658, 2017.

18. Wan Y, Vagenas D, Salazar C, Kenny L, Perry C, Calvopiña D and Punyadeera C: Salivary miRNA panel to detect HPV-positive and HPV-negative head and neck cancer patients. Oncotarget 8 : 99990-100001, 2017.

19. Zeng Y, Zhu J, Shen D, Qin H, Lei Z, Li W, Huang JA and Liu Z: Repression of Smad4 by miR-205 moderates TGF- $\beta$-induced epithelial-mesenchymal transition in A549 cell lines. Int J Oncol 49: 700-708, 2016.
20. Voorneveld PW, Kodach LL, Jacobs RJ, Liv N, Zonnevylle AC, Hoogenboom JP, Biemond I, Verspaget HW, Hommes DW, de Rooij K, et al: Loss of SMAD4 alters BMP signaling to promote colorectal cancer cell metastasis via activation of Rho and ROCK. Gastroenterology 147: 196-208, 2014.

21. Ormanns S, Haas M, Remold A, Kruger S, Kruger S, Holdenrieder S, Kirchner T, Heinemann V and Boeck S: The impact of SMAD4 loss on outcome in patients with advanced pancreatic cancer treated with systemic chemotherapy. Int J Mol Sci 18: E1094, 2017

22. Wang Z, Yang J, Di J, Cui M, Xing J, Wu F, Wu W, Yang H, Zhang C, Yao Z, et al: Downregulated USP3 mRNA functions as a competitive endogenous RNA of SMAD4 by sponging miR-224 and promotes metastasis in colorectal cancer. Sci Rep 7: 4281, 2017.

23. Lee CC, Yang WH, Li CH, Cheng YW, Tsai CH and Kang JJ: Ligand independent aryl hydrocarbon receptor inhibits lung cancer cell invasion by degradation of Smad4. Cancer Lett 376: 211-217, 2016

24. Park JW, Jang SH, Park DM, Lim NJ, Deng C, Kim DY, Green JE and Kim HK: Cooperativity of E-cadherin and Smad4 loss to promote diffuse-type gastric adenocarcinoma and metastasis. Mol Cancer Res 12: 1088-1099, 2014.

25. Phuah NH, Azmi MN, Awang $\mathrm{K}$ and Nagoor NH: Down-regulation of microRNA-210 confers sensitivity towards 1'S-1'-acetoxychavicol acetate (ACA) in cervical cancer cells by targeting SMAD4. Mol Cells 40: 291-298, 2017.

26. Cheng H, Fertig EJ, Ozawa H, Hatakeyama H, Howard JD, Perez J, Considine M, Thakar M, Ranaweera R, Krigsfeld G and Chung CH: Decreased SMAD4 expression is associated with induction of epithelial-to-mesenchymal transition and cetuximab resistance in head and neck squamous cell carcinoma. Cancer Biol Ther 16: 1252-1258, 2015.

27. Liu N, Yu C, Shi Y, Jiang J and Liu Y: SMAD4 expression in breast ductal carcinoma correlates with prognosis. Oncol Lett 10: 1709-1715, 2015

28. Hernanda PY, Chen K, Das AM, Sideras K, Wang W, Li J, Cao W, Bots SJ, Kodach LL, de Man RA, et al: SMAD4 exerts a tumor-promoting role in hepatocellular carcinoma. Oncogene 34: 5055-5068, 2015

29. Ling H, Pickard K, Ivan C, Isella C, Ikuo M, Mitter R, Spizzo R, Bullock M, Braicu C, Pileczki V, et al: The clinical and biological significance of MIR-224 expression in colorectal cancer metastasis. Gut 65: 977-989, 2016.

30. Cheng D, Zhao S, Tang H, Zhang D, Sun H, Yu F, Jiang W, Yue B, Wang J, Zhang M, et al: MicroRNA-20a-5p promotes colorectal cancer invasion and metastasis by downregulating Smad4. Oncotarget 7: 45199-45213, 2016.

31. Haeger SM, Thompson JJ, Kalra S, Cleaver TG, Merrick D, Wang XJ and Malkoski SP: Smad4 loss promotes lung cancer formation but increases sensitivity to DNA topoisomerase inhibitors. Oncogene 35: 577-586, 2016.

32. Liu L, Nie J, Chen L, Dong G, Du X, Wu X, Tang Y and Han W: The oncogenic role of microRNA-130a/301a/454 in human colorectal cancer via targeting Smad4 expression. PLoS One 8: e55532, 2013

33. Osugi J, Muto S, Matsumura Y, Higuchi M, Suzuki H and Gotoh M: Prognostic impact of the high-sensitivity modified Glasgow prognostic score in patients with resectable non-small cell lung cancer. J Cancer Res Ther 12: 945-951, 2016.

This work is licensed under a Creative Commons Attribution-NonCommercial-NoDerivatives 4.0 International (CC BY-NC-ND 4.0) License. 